\title{
Development of a Risk-Scoring Tool to Determine Appropriate Level of Care in Acute Bacterial Skin and Skin Structure Infections in an Acute Healthcare Setting
}

Kimberly C. Claeys · Evan J. Zasowski · Abdalhamid M. Lagnf •

Noor Sabagha · Donald P. Levine · Susan L. Davis · Michael J. Rybak

Received: June 1, 2018 / Published online: September 22, 2018

(C) The Author(s) 2018

\section{ABSTRACT}

Introduction: Acute bacterial skin and skin structure infections (ABSSSIs) represent a large burden to the US healthcare system. There is

Enhanced digital features To view enhanced digital features for this article go to https://doi.org/10.6084/ m9.figshare.7053953.

Electronic supplementary material The online version of this article (https://doi.org/10.1007/s40121018-0212-3) contains supplementary material, which is available to authorized users.

K. C. Claeys

University of Maryland School of Pharmacy,

Baltimore, MD, USA

E. J. Zasowski · A. M. Lagnf · M. J. Rybak ( $₫)$ Anti-Infective Research Laboratory, Wayne State University Eugene Applebaum College of Pharmacy and Health Sciences, Detroit, MI, USA

e-mail: aa1592@wayne.edu

\section{E. J. Zasowski}

University of Houston College of Pharmacy, Houston, TX, USA

N. Sabagha

School of Medicine, Wayne State University,

Detroit, MI, USA

D. P. Levine - M. J. Rybak

Division of Infectious Disease, Wayne State

University School of Medicine, Detroit, MI, USA

S. L. Davis

Wayne State University Eugene Applebaum College of Pharmacy and Health Sciences, Detroit, MI, USA little evidence-based guidance regarding the appropriate level of care for ABSSSIs. This study aimed to develop a prediction model and riskscoring tool to determine appropriate levels of care.

Methods: This was a single-center observational cohort study of adult patients treated for ABSSSIs from 2012 to 2015 at the Detroit Medical Center. The predictive model used to create a novel risk-scoring tool was derived using multinomial regression analysis. The overall accuracy of this tool was compared to the Clinical Resource Efficacy Support Team (CREST) Classification and Standardized Early Warning Score (SEWS) using area-under-thereceiver-operator-curve (AUROC) analysis and Z-statistic.

Results: Final patient disposition was 230 (45.5\%) home from the emergency department (ED), 65 (12.8\%) observation unit (OU), and 211 (41.7\%) initial inpatient. IV antibiotic therapy was used in 358 (70.8\%) patients. CREST and SEWS were not accurate in the determination of ED versus OU disposition [AUROC CREST 0.0.682 (95\% CI 0.640-0.724), AUROC SEWS 0.686 (95\% CI 0.641-0.731)], but performed better in determining $\mathrm{ED} / \mathrm{OU}$ versus inpatient [AUROC CREST $=0.678$ (95\% CI 0.630-0.725), AUROC SEWS 0.693 (95\% CI 0.645-0.740)]. These scores were also not accurate in determining IV versus PO antibiotic therapy [AUROC CREST $=0.586$ (95\% CI 0.530-0.624), AUROC SEWS $=0.630(95 \%$ CI $0.576-0.684)]$. A risk- 
scoring tool ranging from 0 to 10 points was derived incorporating WBC, temperature, site of infection, and past medical history of diabetes, liver disease, PVD, AKI, and/or CKD. The AUROC of the new model was 0.675 (95\% CI 0.611-0.739) ED versus OU, 0.789 (95\% CI 0.748-0.829) ED/OU versus inpatient, and 0.742 (95\% CI 0.694-0.789) IV versus oral antibiotics. The new score had a significantly higher AUROC compared to both the CREST and SEWS for determining ED/OU versus inpatient $(p<0.001)$.

Conclusion: Prediction models based on patient risk may be useful for determining appropriate level of care during for ABSSSIs. While the prediction model demonstrated moderate to high levels of correlation with patient level of care, further validation of a prospective cohort of patients is warranted.

Keywords: Acute bacterial skin and skin structure infection; Emergency department; Observation unit; Patient disposition; Predictive analytics

\section{INTRODUCTION}

Acute bacterial skin and skin structure infections (ABSSSIs) represent a large burden to acute care hospitals. In the United States, these infections account for approximately 2.27 million emergency department (ED) visits, and over 800,000 hospital admissions annually [1-3]. According to the Healthcare Cost and Utilization Project National Inpatient Sample, hospital encounters for ABSSSIs are on the rise, associated with a mean adjusted cost of $\$ 9388$ USD per visit [4]. To ease the increased burden and costs of ABSSSIs, ED and observation units (OUs) are used to manage patients likely to be discharged home within $24 \mathrm{~h}$ of initial care $[5,6]$. Currently, however, there is a lack of consensus to establish which patients benefit most from ED/OU stay versus inpatient admission [7-11].

Several severity scoring systems have been developed to aid in the determination of patient acuity and proper level of care in the management of ABSSSIs: these include the
Eron/CREST (Clinical Resource Efficacy Support Team) Classification, the modified CREST using the Standardized Early Warning Severity Score (SEWS), the Ki and Rosenstein Score, and the Wilson Score [12-16]. The Eron consensus criteria are used primarily in the UK as part of the CREST Guidelines for The Management of Cellulitis in Adults and are based on expert opinion $[13,15]$. This severity classification uses patient past medical history and systemic signs and symptoms of infection to aid in the decision of site of care and determination of most appropriate antibiotic therapy. Marwick et al. used a modified version of the CREST classification together with an early patient acuity score and SEWS and demonstrated that CREST often led to over-treated patients $[12,17]$. These scoring systems, however, have not been robustly validated and none have been utilized to determine ED versus OU status.

Evidence-based guidance is needed regarding which patients should be considered for short stay in the $\mathrm{ED} / \mathrm{OU}$ versus inpatient setting before discharge. There is a need to better define the most appropriate level of care and antibiotic therapy for patients with ABSSSIs in order to maximize clinical outcomes while minimizing cost burden. Previous studies with clinical decision rules and risk-scoring tools, such as the CREST Classification, have demonstrated that a combination of patient co-morbidities and presenting physiological parameters is likely key to aiding in the determination of optimal patient management. This study sought to derive a predictive model and severity scoring system to aid in assessing patient acuity and predicting most appropriate levels of care, locations and antibiotic therapy, in order to decrease unnecessary hospitalizations, decrease costs of care, and ultimately improve patient outcomes.

\section{METHODS}

\section{Study Population, Study Period, Setting}

This was a retrospective observational cohort study of adult patients (18-99 years of age) initially treated in EDs of the Detroit Medical 
Center (DMC) for the primary diagnosis of ABSSSI for index/initial visit from April 2012 to 2015. ABSSSIs were diagnosed and categorized based on physician discretion and Food and Drug Administration criteria [18]. To be eligible for study inclusion, patients were required to present with at least three of the following local signs/symptoms: pain, tenderness, swelling erythema, warmth, drainage/discharge, induration, and lymph node swelling/tenderness. Patients were excluded if there was clinical concern for gas gangrene, necrotizing fasciitis, osteomyelitis, hardware-related infections, or complicated bacteremia. Additional exclusion criteria were: open burn $>30 \%$ of total body surface area, life expectancy $\leq 2$ months, pregnant or nursing, or prisoner at time of treatment. Patients who were "inappropriately treated" were also excluded from derivation of the predictive model and risk-scoring tool due to the potential for misclassification bias affecting the final model's performance. Inappropriate treatment was determined to be: $\mathrm{ED}$ treatment $>6 \mathrm{~h}$, OU treatment $>24 \mathrm{~h}$, inpatient admission $<24 \mathrm{~h}$, or ED revisit within $96 \mathrm{~h}$ of initial treatment for ABSSSI $[19,20]$. The setting of the study was two acute care trauma institutions (Detroit Receiving Hospital and Harper University Hospital) and two community-based hospitals (Sinai-Grace Hospital and Huron Valley Sinai Hospital) of the DMC, one of the largest healthcare providers in Southeast Michigan. This study was approved by the institutional review boards (IRB) of Wayne State University granting a waiver of informed consent. All procedures performed involving human participants were in accordance with the ethical standards of the IRB and with the 1964 Helsinki declaration and its later amendments or comparable ethical standards.

\section{Methods for Data Collection and Clinical Assessment}

Patient data collection from electronic medical health records included the following metrics: patient demographics, type of insurance (Medicare/Medicaid/private/mixed), co-morbid conditions, antibiotic history (previous 60 days) as available, hospitalization history (previous 180 days) as available, previous history of ABSSSI as available (previous year), type of ABSSSI (cellulitis, abscess, wound), and criteria for systemic inflammatory response syndrome (SIRS). Additionally, patients' initial antibiotic(s), discharge antibiotic prescription(s), and non-pharmacological treatments (debridement/ incision and drainage/amputation) were documented. Patient outcomes, including 96-h ED revisit, final hospital location, time receiving care (hours) in the ED/OU/inpatient setting, 7-day, 14-day and 30-day infection-related ED revisits and/or hospital admission were also recorded. Location of care was divided into ED, $\mathrm{OU}$, and inpatient (floor and intensive care unit). Higher location of care was denoted inpatient $>\mathrm{OU}>\mathrm{ED}$. Two severity-scoring systems were calculated and compared for this patient population: the Enron/CREST Classification and SEWS, which have been previously published [12, 13, 15, 17].

\section{Statistical Analysis}

Descriptive statistics were summarized using means (standard deviations) or medians [interquartile range (IQR)], as applicable. Continuous variables were compared between $\mathrm{ED}, \mathrm{OU}$, and inpatient using one-way analysis of variance (ANOVA) or Kruskal-Wallis ANOVA, with modified Bonferroni correction, as applicable. Categorical variables, such as patient demographics and clinical characteristics at presentation, were compared using $\chi^{2}$ test or the Fisher's exact test, as applicable. A series of multinomial logistic regression models were run to select independent predictors of the need for higher location of care. Variables with significance on univariate analysis $(p<0.1)$ or determined to be of clinical significance a priori were entered into the models. Variables selected to be included in the final model were determined using Akaike Information Criterion [21]. The final regression model was assessed using the Hosmer-Lemeshow goodness-of-fit test [22]. A bootstrapping algorithm with replacement was used for internal validation. Coefficients from the final regression model were standardized to 
a weight-proportional integer value in order to be included in the scoring tool. Area-under-thereceiver operator curve (AUROC) analysis, or the $c$-statistic, was used to determine overall accuracy of the newly derived scoring tool and the $Z$-test was used to compare the new tool to the CREST Classification and SEWS [23]. The modified CREST, incorporating SEWS, was unable to be included as zero patients in the entire cohort presented with SEWS $\geq 4$ [24]. Statistical analysis was performed using IBM SPSS v.23.0 (IBM, Armonk, NY, USA) with the exception of AUROC comparisons, which were completed in SAS v.9.4 (SAS Institute, Cary, NC, USA).

\section{RESULTS}

\section{Study Population Characteristics}

During the study period, 1240 patients were screened. Exclusions were: age $<18$ years $(n=6)$; underlying bone/joint infection ( $n=258) ;$ suspicion of gangrene/necrotizing fasciitis $(n=104)$; presence of prosthetic hardware $(n=75)$; missing data $(n=63)$; repeat visit during study period $(n=242)$; and odontogenic infection $(n=9)$. Among patients meeting inclusion $(n=665), 506$ patients were "appropriately treated" and retained for analysis and development of the predictive model and scoring tool (Fig. 1). Among the patients included, $230(45.5 \%)$ were treated in the ED, $65(12.8 \%)$ in the OU, and $211(41.7 \%)$ were admitted for inpatient management. The median age of the entire study population was 43 years (IQR

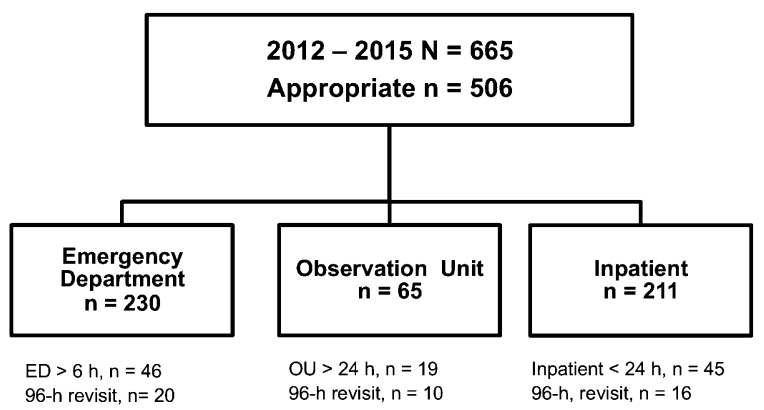

Fig. 1 Flow diagram of patient inclusion
31-55 years) and the majority of patients were black $(n=534,81.7 \%)$; these demographics reflect the patient population seen in the metropolitan Detroit area. Patients presenting with abscess were most common $(n=275$, $54.3 \%)$, followed by cellulitis $(n=182,35.9 \%)$, and finally wound infections ( $n=49,9.7 \%)$.

Patient demographics and clinical characteristics by location of care are summarized in Table 1. Patients treated and discharged from the ED were significantly more likely to present with an abscess, while wound infections were more likely to be admitted for inpatient management. Increasing age and increasing Charlson Comorbidity Score both led to patients being more likely to be admitted. Patients with a past medical history of diabetes, chronic kidney disease, or peripheral vascular disease were also more likely to be admitted for inpatient management. Patient clinical presentation varied significantly by location of care. In particular, all four metrics within the SIRS criteria were associated with OU stay or inpatient admission. Alterations in body temperature $\left(\leq 36.0\right.$ or $\left.>38.0^{\circ} \mathrm{C}\right)$ and/or white blood cell count $\left(<4000\right.$ or $>12,000$ cells $\left./ \mathrm{m}^{3}\right)$ were significantly associated with inpatient admission. Patients presenting with ABSSSI of the arm or head/neck were more likely to be directly from the ED, while patients with an ABSSSI of the foot or leg were associated with OU or inpatient stay. Patient healthcare exposure history was variable, with the notable exception that there was no difference based on reported failure of outpatient antibiotics.

\section{Multivariable Model and Risk-Score Development for Patient Location of Care}

Numerous variables met the a priori inclusion criteria of significance at a level of $p<0.1$ on univariate analysis. Variables that were considered to be co-linear or not externally valid were not included in the model. As an example, in the current cohort, infections of the head/neck were significantly more likely to be treated in the ED, while previous literature has associated infections of the head/neck with inpatient admission [7, 10]. The initial multinomial 
Table 1 Baseline demographics and clinical presentation by setting of care

\begin{tabular}{|c|c|c|c|c|}
\hline Characteristics & $\mathrm{ED}(n=230)$ & OU $(n=65)$ & Inpatient $(n=211)$ & $p$ value \\
\hline Age, years $(\mathrm{IQR})^{\mathrm{a}}$ & $38(27-49)$ & $47(38-53)$ & $48(37-58)$ & $<0.001$ \\
\hline Charlson Comorbidity Score, median (IQR) & $0(0-2)$ & $1(0-2)$ & $2(1-4)$ & $<0.001$ \\
\hline \multicolumn{5}{|l|}{ Type of ABSSSI } \\
\hline Cellulitis & $79(34.73)$ & $30(46.1)$ & $73(34.6)$ & 0.186 \\
\hline Abscess & $133(57.8)$ & $33(50.7)$ & $109(51.7)$ & $<0.001$ \\
\hline Wound infection & $18(7.8)$ & $2(3.1)$ & $29(13.7)$ & 0.017 \\
\hline \multicolumn{5}{|l|}{ Comorbidities } \\
\hline Diabetes mellitus & $32(13.9)$ & $12(18.5)$ & $71(33.6)$ & $<0.001$ \\
\hline Obesity (BMI $\geq 30 \mathrm{~kg} / \mathrm{m}^{2}$ ) & $94(40.9)$ & $24(36.9)$ & $105(49.8)$ & 0.079 \\
\hline Chronic kidney disease & $6(2.6)$ & $2(3.1)$ & $20(9.5)$ & 0.005 \\
\hline Liver disease & $2(0.9)$ & $3(4.6)$ & $13(6.2)$ & 0.010 \\
\hline Injection drug use & $11(4.8)$ & $5(7.7)$ & $25(11.8)$ & 0.025 \\
\hline Peripheral vascular disease & $2(0.9)$ & $5(7.7)$ & $27(12.8)$ & $<0.001$ \\
\hline \multicolumn{5}{|l|}{ SIRS at presentation } \\
\hline Temperature $\left(>38.0\right.$ or $\left.\leq 36.0^{\circ} \mathrm{C}\right)$ & $36(15.7)$ & $18(27.7)$ & $86(40.8)$ & $<0.001$ \\
\hline $\mathrm{WBC}>12,000$ or $<4000$ & $22(9.6)$ & $16(24.6)$ & $90(42.7)$ & $<0.001$ \\
\hline Heart rate $\geq 90$ beats $/ \mathrm{min}$ & $92(40.0)$ & $38(58.5)$ & $128(60.7)$ & $<0.001$ \\
\hline Respiratory rate $\geq 20 / \mathrm{min}$ & $3(1.3)$ & $6(9.2)$ & $15(7.1)$ & 0.003 \\
\hline Acute kidney injury ${ }^{\mathrm{b}}$ & $9(3.9)$ & $2(3.1)$ & $20(9.6)$ & $<0.001$ \\
\hline \multicolumn{5}{|l|}{ Primary location of ABSSSI } \\
\hline Arm & $47(20.4)$ & $10(15.4)$ & $20(9.5)$ & 0.006 \\
\hline Hand & $16(7.0)$ & $13(20.0)$ & $26(12.3)$ & 0.008 \\
\hline Foot & $12(5.2)$ & $4(6.2)$ & $25(11.8)$ & 0.032 \\
\hline Leg & $35(15.2)$ & $17(26.2)$ & $64(30.3)$ & 0.001 \\
\hline Torso & $77(33.5)$ & $16(24.6)$ & $64(30.3)$ & 0.379 \\
\hline Head/neck & $43(18.7)$ & $5(7.7)$ & $12(5.7)$ & $<0.001$ \\
\hline Prior history of ABSSSI, past year & $49(21.3)$ & $13(20.0)$ & $57(27.0)$ & 0.285 \\
\hline Prior hospitalization, 180 days & $16(7.0)$ & $6(9.2)$ & $36(17.1)$ & 0.003 \\
\hline Prior healthcare exposure ${ }^{\mathrm{c}}, 30$ days & $44(19.1)$ & $16(24.6)$ & $51(24.2)$ & 0.378 \\
\hline Prior antibiotics, 90 days & $34(14.8)$ & $8(12.3)$ & $26(12.3)$ & 0.721 \\
\hline
\end{tabular}


Table 1 continued

\begin{tabular}{lcccc}
\hline Characteristics & ED $(\boldsymbol{n}=\mathbf{2 3 0})$ & OU $(\boldsymbol{n}=\mathbf{6 5})$ & Inpatient $(\boldsymbol{n}=\mathbf{2 1 1})$ & $\boldsymbol{p}$ value \\
\hline Failed outpatient antibiotics & $14(6.1)$ & $2(3.1)$ & $15(7.1)$ & 0.495 \\
\hline
\end{tabular}

ABSSSI acute bacterial skin and skin structure infection, BMI body mass index, IQR interquartile range, SIRS systemic inflammatory response syndrome, $W B C$ white blood cell count

${ }^{a}$ Kruskal-Wallis one-way analysis of variance test

b A minimum of two or three consecutive documented increases in serum creatinine concentrations (defined as an increase of $0.5 \mathrm{mg} / \mathrm{dL}$ ) or $\geq 50 \%$ increase from baseline

${ }^{c}$ Prior healthcare exposure $=$ hospitalization $>48$ h; ED visit; hemodialysis or wound care clinic; nursing home resident

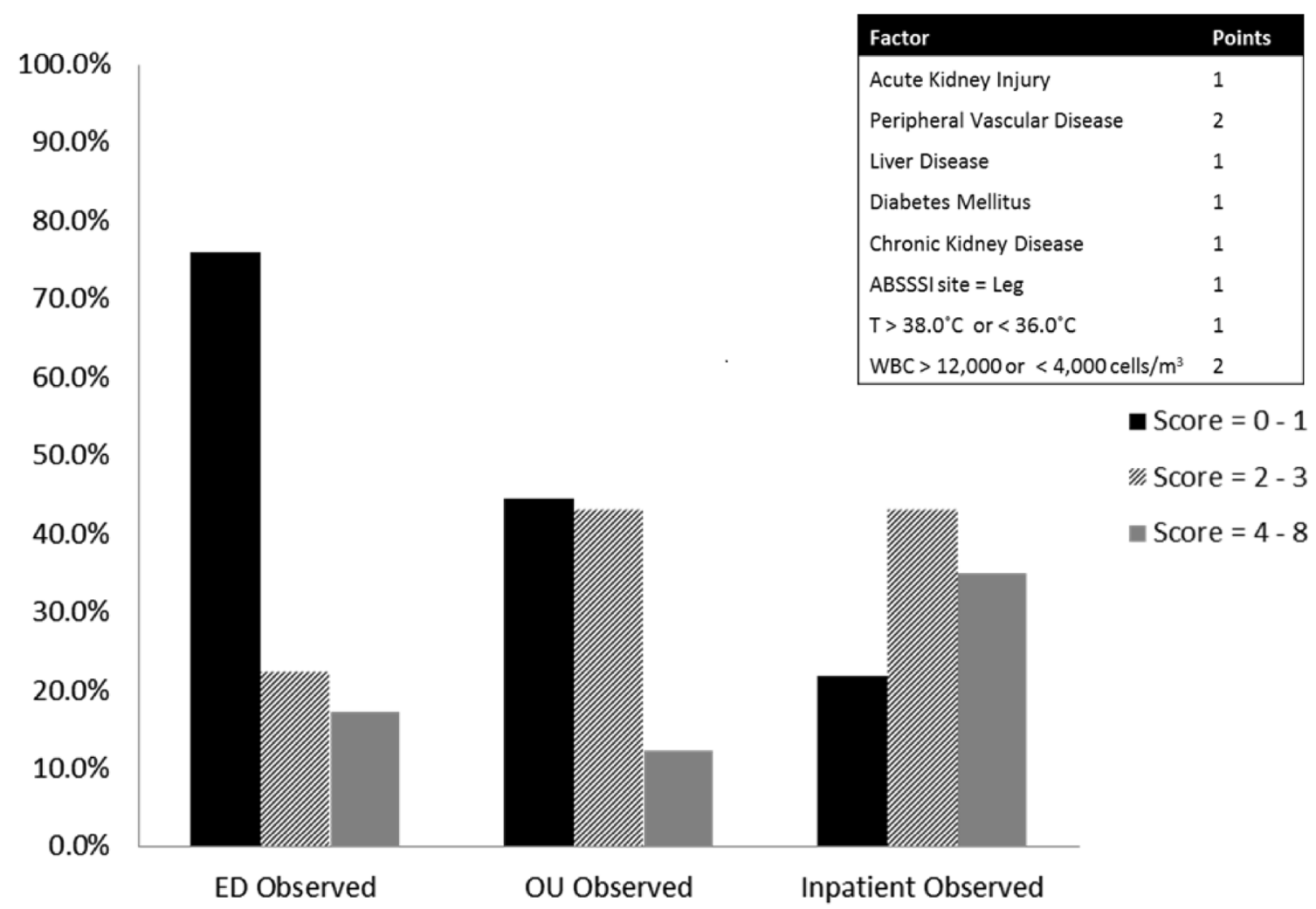

Fig. 2 Risk-scoring tool and score distribution by location of care

regression model included: past medical history of diabetes, peripheral vascular disease, liver disease, chronic kidney disease, acute kidney injury, all four SIRS criteria, and primary ABSSSI location of hand or leg. After a series of multinomial regression models, the final model consisted of eight variables: acute kidney injury, chronic kidney disease, peripheral vascular disease, diabetes, liver disease, ABSSSI site of leg, temperature alteration $\left(>38.0\right.$ or $\left.\leq 36.0^{\circ} \mathrm{C}\right)$, and WBC alteration $(>12,000$ or $<4000$ cells/ $\mathrm{m}^{3}$ ). The distribution of variation derived from bootstrapping confirms internal validity of the comparator group, in this instance, ED (Supplemental Table 1).

Using the coefficients of the final regression model, a scoring tool was developed that ranged from 0 to 10 points (Fig. 2); the higher the score, 


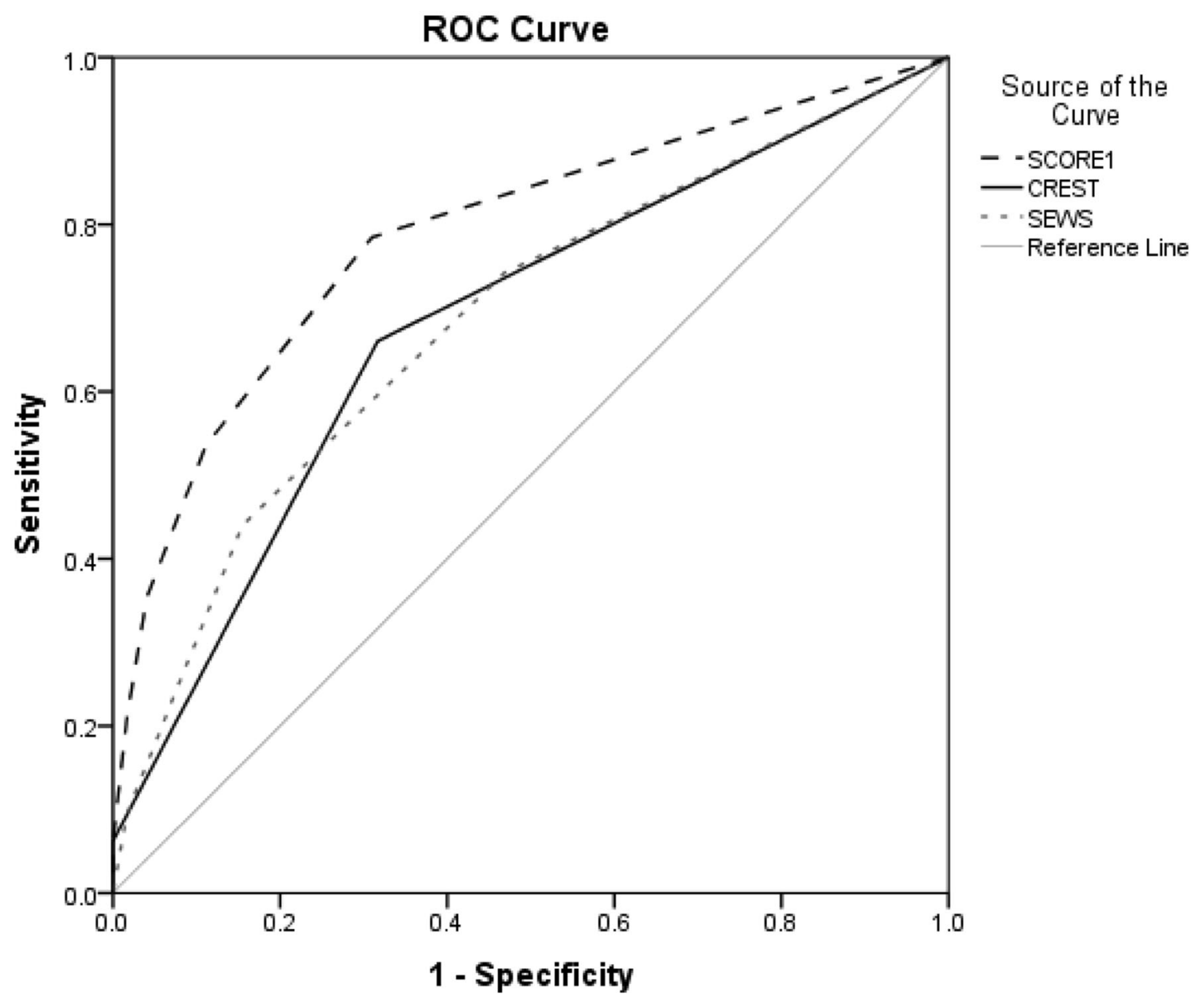

Fig. 3 Overall accuracy of scoring tool derived from the predictive model ED/OU versus Inpatient

Table 2 ROC association statistics (ED/OU vs. inpatient)

\begin{tabular}{llll}
\hline ROC model & AUC & SE & 95\% confidence interval \\
\hline CREST & 0.6817 & 0.0213 & $(0.6400,0.7235)$ \\
SEWS & 0.6861 & 0.0229 & $(0.6412,0.7309)$ \\
SCORE1 & 0.7886 & 0.0207 & $(0.7480,0.8291)$ \\
\hline
\end{tabular}

The curve of CREST is not significantly different from the curve of SEWS $(p=0.8830)$; the curve of CREST is significantly different from the curve of SCORE1 $(p<0.0001)$; the curve of SEWS is significantly different from the curve of SCORE1 $(p<0.0001)$

the more likely the patient was admitted for inpatient management of ABSSSI. At a score of
$0,76.1 \%$ of patients were treated and released directly from the ED. The overall accuracy (AUROC) of the new severity-scoring tool to determine patient location of care was compared to the CREST Classification and SEWS score (Fig. 3). Overall, none of the three scoring tools were able to accurately predict $\mathrm{ED}$ versus OU. The AUROC for the CREST Classification was 0.527 (95\% CI 0.460-0.594), SEWS score was 0.609 (95\% CI $0.545-0.673$ ), and the newly developed scoring tool was 0.675 (95\% CI 0.611-0.739). All three scores were better able to predict ED/OU versus inpatient disposition, and the newly developed model was able to outperform previous scores (Tables 2, 3). The AUROC for the CREST Classification was 0.682 (95\% CI 0.640-0.724) and the SEWS score was 0.686 (95\% CI 0.641-0.731). For the newly 
Table 3 ROC contrast test results (ED/OU vs. inpatient)

\begin{tabular}{ccccc}
\hline Contrast & ESTIMATE & SE & $\begin{array}{l}\text { 95\% } \\
\text { confidence } \\
\text { interval }\end{array}$ & $p$ value \\
& & & & \\
\hline SEWS vs & 0.00435 & 0.0296 & $(-0.0536$, & 0.8830 \\
CREST & & & $0.0623)$ & \\
SCORE1 & 0.1068 & 0.0245 & $(0.0587$, & $<0.0001$ \\
vs & & & $0.1549)$ & \\
CREST & & & & \\
SCORE1 & 0.1025 & 0.0214 & $(0.0606$, & $<0.0001$ \\
vs & & & $0.1443)$ & \\
SEWS & & & & \\
\hline
\end{tabular}

The curve of CREST is not significantly different from the curve of SEWS $(p=0.8830)$; the curve of CREST is significantly different from the curve of SCORE1 $(p<0.0001)$; the curve of SEWS is significantly different from the curve of SCORE1 $(p<0.0001)$

developed model, the AUROC was 0.789 (95\% CI 0.748-0.829). The CREST Classification and SEWS score were not significantly different $(p=0.883)$; however, the newly developed model had a significantly higher AUROC compared to both the CREST Classification and the SEWS score $(p<0.0001)$ (Tables 2, 3; Fig. 3).

\section{Antibiotic Therapy for ABSSSI}

Choice of Initial antibiotic therapy for the treatment of ABSSSI varied significantly based on location of care (Table 4). Unsurprisingly, initial oral antibiotics were prescribed more often in patients treated and discharged directly from the $\mathrm{ED}$, with over $50 \%$ of regimens being oral, compared to over $80 \%$ in patients treated in the OU or inpatient. MRSA coverage was more common in the ED, regardless of type of underlying ABSSSI (cellulitis vs. wound versus abscess); this was largely driven by prescriptions for clindamycin in either IV or oral formulation. Vancomycin was frequently employed in the OU and inpatient setting regardless of type of ABSSSI. Narrower spectrum agents such as cefazolin or nafcillin were only used in two patients. Gram-negative coverage was also significantly more common in patients treated in the OU or inpatient. Overly broad antibiotic coverage, defined as receipt of Gram-negative coverage, was most common among CREST Class III (50\%), followed by CREST Class II (30.6\%).

According to the CREST Classification, those in CREST I should be treated outpatient with oral antibiotics. In this patient population, however, only $32.1 \%$ of CREST I patients were treated initially with oral agents. CREST II patients were treated with oral antibiotics in $19.6 \%$ of cases, and zero patients in CREST III received initial oral therapy. Overall accuracy of the CREST Classification for determining IV versus oral initial antibiotic therapy was low (AUROC CREST $=0.586$ (95\% CI 0.530-0.624). Within the newly developed scoring tool, $42.6 \%$ of patients with a score of $0-1 ; 10.6 \%$ with a score of $2-3$; and $8.1 \%$ of patients with a score of $4-8$ were prescribed oral antibiotic therapy initially. The newly developed scoring tool performed marginally better in terms of overall accuracy for determination of IV versus oral empiric therapy [AUROC $=0.742(95 \%$ CI 0.694-0.789)].

\section{DISCUSSION}

Although ABSSSIs are common in the United States, and the Infectious Disease Society of America guidelines have been recently updated, there are still significant gaps in our understanding of optimal management [13]. In 2003, Eron et al. created the consensus-based criteria that would later be utilized in the CREST guidelines for the management of cellulitis in adults in the United Kingdom [13, 15]. This classification scheme aimed to guide appropriate patient disposition and antibiotic therapy. Since then, several studies have demonstrated that the CREST Classification tends to over-estimate level of care. In an analysis of 533, 263 ED visits for skin infections in 2003 from the Premier Hospital Database by Sulham et al., $57.85 \%$ of CREST Class II patients and $22.65 \%$ of CREST Class III patients were successfully treated on an outpatient basis with oral antibiotics [25]. Marwick et al. attempted to refine the 
Table 4 Initial and discharge antibiotic therapy for ABSSSI by location of care

\begin{tabular}{lcccc}
\hline & ED $(\boldsymbol{n}=\mathbf{2 3 0})$ & OU $(\boldsymbol{n}=\mathbf{6 5})$ & Inpatient $(\boldsymbol{n}=\mathbf{2 1 1})$ & $\boldsymbol{p}$ value \\
\hline Initial antibiotics & & & & $<0.0001$ \\
Intravenous route & $103(44.8)$ & $53(81.5)$ & $202(96.2)$ & $<0.0001$ \\
Clindamycin & $181(78.7)$ & $16(24.6)$ & $66(31.3)$ & $<0.0001$ \\
Vancomycin & $14(6.1)$ & $29(44.6)$ & $89(42.2)$ & 0.030 \\
Ampicillin-sulbactam & $24(10.4)$ & $15(23.1)$ & $29(13.7)$ & 0.013 \\
MRSA coverage $^{\mathrm{a}}$ & $195(84.8)$ & $45(69.2)$ & $162(77.5)$ & $<0.0001$ \\
Gram-negative $^{\mathrm{b}}$ & $32(11.9)$ & $25(23.6)$ & $68(23.4)$ & \\
Discharge antibiotics & & & & 0.005 \\
Clindamycin $^{\text {TMP/SMX }}$ & $150(65.2)$ & $20(30.8)$ & $68(32.2)$ & 0.464 \\
Cephalexin & $33(14.3)$ & $9(10.6)$ & $40(18.9)$ & 0.337 \\
Combination & $31(13.4)$ & $10(11.8)$ & $16(8.7)$ & 0.336 \\
\hline
\end{tabular}

$I Q R$ interquartile range, MRSA methicillin-resistant $S$. aureus, TMP/SMX trimethoprim/sulfamethoxazole

a MRSA coverage: vancomycin, clindamycin, ceftaroline, linezolid, daptomycin, TMP/SMX

b Gram-negative coverage: beta-lactam/beta-lactamase inhibitor, ceftriaxone, cefepime

CREST Classification and decrease clinical ambiguity by adding the SEWS score. The SEWS score has been significantly linked to increased hospital length of stay, need for intensive care, and overall mortality and, as such, seems like an optimal addition to the CREST Classification [26]. In a retrospective analysis of 205 patients, Marwick et al. determine that approximately $26 \%$ of patients received appropriate care based on this modified CREST Classification; however, this did not negatively impact overall clinical outcomes [12]. They sought to further validate this tool through a prospective study, but were unable to do so secondary to limited sample size [17]. Recently, Hashem et al. conducted a single-center retrospective assessment of the CREST Classification in 200 patients in the United States [24]. The authors found, similar to Marwick et al., that the CREST Classification resulted in significant over-treatment, with 63\% of CREST Class I patients being treated with intravenous (IV) antibiotics when oral options were recommended as sufficient or overly broad-spectrum antibiotic therapy was used. Through these studies, it has become clear that, although the CREST Classification is potentially flawed, using a clinical decision support tool does have the potential to optimize patient care in the management of ABSSSIs.

Over the years, OUs or clinical decision support units have gained popularity for the treatment of patients and conditions that do not necessarily need several days of inpatient management but cannot be successfully treated purely on an outpatient basis. OUs can been advantageous to healthcare systems through decreasing unnecessary admissions, improving efficiency and patient satisfaction, and decreasing costs of care $[5,19]$. Cost avoidance, through outpatient management versus the fixed payment system used for inpatient management, is a main driver for changes in how ABSSSIs are managed [8, 27]. The American College of Emergency Physicians recommends evidence-based guidelines to establish patients best suited to have successful care in the OU [19]. In their policy resource and education paper, however, it is clear that this is easier said than done in the case of ABSSSIs. Recently, Lodise et al. examined the economic impact of 
potentially avoidable admissions in patients with ABSSSIs [28]. In a large database analysis of over 120,000 inpatient cases, those without lifethreating conditions or systemic signs and symptoms represented the largest group $(n=100,267)$. Additionally, more than half of these patients presented with a Charlson Comorbidity Score of zero. The care of these patients was associated with a mean cost of \$5851 (SD \$6756) USD per patient, which represents a large and potentially avoidable economic burden.

Several studies have specifically aimed to determine which past medical history and clinical characteristics in patients presenting to the ED with ABSSSIs result in either the need to upgrade care to inpatient admission or lead to overall failure of ED/OU care. These studies fail to provide a strong consensus. In a retrospective analysis by Sabbaj et al., the researchers attempted to develop a clinical decision rule to determine the need for more than $24 \mathrm{~h}$ of acute care. Although they were unable to develop a high sensitivity rule, a temperature $>37.8^{\circ} \mathrm{C}$ was associated with an odds of 2.91 (95\% CI 1.65-5.12) of requiring more than $24 \mathrm{~h}$ of care [9]. A study by Volz et al. also demonstrated that an elevation in body temperature at presentation was associated with requiring more than $24 \mathrm{~h}$ of care at an odds ratio of 2.5 (95\% CI 1.1-5.5) [7]. Additionally, a retrospective study by Shrock et al. found that an elevation in WBC $>15,000$ cells $/ \mathrm{m}^{3}$ was associated with a four-fold odds (OR $=4.06,95 \%$ CI 1.53-10.74) of requiring greater than $24 \mathrm{~h}$ of care [10]. The currently derived predictive model encompasses similar metrics in terms of physiological parameters, with the addition of several comorbid conditions. This highlights the importance of considering a combination of factors when decided on appropriate treatment for ABSSSI extending beyond past medical history or size of lesion. The clinical utility of these models outside of the patient populations they have been studied in has not been fully explored. External generalizability of these tools may prove to be lacking; however, they do provide a framework for which other sites can build off to develop their own risk-scoring tools and clinical pathways in order to decrease inappropriate admissions or the use of intravenous antibiotics in ABSSSIs.

Although the current study demonstrated a small improvement in predictive ability with the newly derived risk score (higher AUROC), there are several limitations that must be noted. With the retrospective, single-centered nature of the study, there is likely recording and selection bias associated with data collection as well as limitations related to external generalizability. Only metrics available in the electronic medical record could be used to derive the model. The size of the affected area or lesion was not available for the majority of patients, which may confound results. For instance, the site of ABSSSI on the leg remained in the predictive model; however, this may represent the size of the lesion as opposed to the actual site. Additionally, practice patterns for our ED/OU may be different compared with other sites and may be altered secondary to factors not related to metrics in the electronic medical record (i.e. bed availability). Also, 96-h ED revisit was used to determine which patients were potentially inappropriately treated instead of the traditional 7 days. The DMC is only one of several large academic medical centers in the Detroit metropolitan area. Using a 96-h ED revisit endpoint is meant to help capture those patients likely presenting with a failure of initial treatment, as opposed to a re-infection, but revisits are likely missed. Importantly, there may be misclassification bias in the analysis as patients treated at a higher level of care may have improved clinical outcomes despite not necessarily needing this higher level of care. Additionally, there is no way to account for inter-patient variability in prescribing practices as there is no gold standard for ABSSSI management to reference. This has potential implications to using this scoring tool outside of the population derived, especially without validation or in populations that were not included in the initial study [29].

\section{CONCLUSION}

The current study highlights opportunities to improve resource utilization and overall clinical 
outcomes in patients with ABSSSIs, and represents an initial step towards a clinical decision support tool. The current model also reinforces previous literature wherein it appears that physiological parameters and comorbid conditions interplay in the determination of appropriate patient disposition. In the future, a similar analysis with a large multicenter database would be warranted to improve external validity of the current predictive model and derived scoring tool. Ideally, this model would then be validated in a prospective, multicenter study in order to improve external validity and overall accuracy, as any retrospective analysis will experience similar limitations to those encountered in this current study. This model can serve as a proof-in-concept blueprint for developing a single-center risk scoring tool and clinical pathway that can aid in decreasing inappropriate resource utilization (i.e. admissions, IV antibiotics) in the management of ABSSSIs. Certain components, along with previously published literature, can assist investigators in deciding upon potentially relevant risk factors. The methods used in this study can also be extrapolated to assist a healthcare site in developing a similar tool that may use different risk factors based on their different patient populations. The results of this study highlight the importance of evaluating the patients with ABSSSIs with respect to not just the immediate clinical picture but also to past medical history.

\section{ACKNOWLEDGEMENTS}

Additional statistical analysis for comparison of ROC curves provided by Hyunuk Seung, MS, Research Analyst SR, Department of Pharmacy Practice and Science, University of Maryland School of Pharmacy.

Funding. No funding or sponsorship was received for this study or publication of this article

Authorship. All named authors meet the International Committee of Medical Journal
Editors (ICMJE) criteria for authorship for this article, take responsibility for the integrity of the work as a whole, and have given their approval for this version to be published.

Disclosures. Kimberly C. Claeys has served as on the advisory board for Milenta Therapeutics. Susan L. Davis has served on advisory boards for Allergan, The Medicines Company, and Zavante and speaker's bureau for Allergan. Donald P. Levine served on the advisory board for Novartis, Contrafect, Melinta Therapeutics, and The Medicines Company, and speaker's bureau for Allergan and Merck. Michael J. Rybak received research support, provided lectures or was a consultant for: Allergan, Bayer, Cempra, Merck, The Medicine Company, Sunovian, Theravance. Evan J. Zasowski, Abdalhamid M. Lagnf, and Noor Sabagha have nothing to disclose.

Compliance with Ethics Guidelines. This protocol was approved by the Wayne State Institutional Review Board, and due to the retrospective nature of the study, a waiver of informed consent was obtained. All procedures performed involving human participants were in accordance with the ethical standards of the IRB and with the 1964 Helsinki declaration and its later amendments or comparable ethical standards.

Data Availability. The datasets during and/ or analyzed during the current study are available from the corresponding author on reasonable request.

Open Access. This article is distributed under the terms of the Creative Commons Attribution-NonCommercial 4.0 International License (http://creativecommons.org/licenses/ by-nc/4.0/), which permits any noncommercial use, distribution, and reproduction in any medium, provided you give appropriate credit to the original author(s) and the source, provide a link to the Creative Commons license, and indicate if changes were made. 


\section{REFERENCES}

1. Mistry RD, Shapiro DJ, Goyal MK, et al. Clinical management of skin and soft tissue infections in the U.S. Emergency departments. West J Emerg Med. 2014;15:491-8.

2. Edelsberg J, Taneja C, Zervos M, et al. Trends in US hospital admissions for skin and soft tissue infections. Emerg Infect Dis. 2009;15:1516-8.

3. Pallin DJ, Egan DJ, Pelletier AJ, et al. Increased US emergency department visits for skin and soft tissue infections, and changes in antibiotic choices, during the emergence of community-associated methicillin-resistant Staphylococcus aureus. Ann Emerg Med. 2008;51:291-8.

4. Kaye KS, Patel DA, Stephens JM, et al. Rising United States hospital admissions for acute bacterial skin and skin structure infections: recent trends and economic impact. PLoS One. 2015;10:e0143276.

5. Wiler JL, Ross MA, Ginde AA. National study of emergency department observation services. Acad Emerg Med. 2011;18:959-65.

6. Roberts R. Management of patients with infectious diseases in an emergency department observation unit. Emerg Med Clin N Am. 2001;19:187-207.

7. Volz KA, Canham L, Kaplan E, et al. Identifying patients with cellulitis who are likely to require inpatient admission after a stay in an ED observation unit. Am J Emerg Med. 2013;31:360-4.

8. Pollack CV Jr, Amin A, Ford WT Jr, et al. Acute Bacterial Skin and Skin Structure Infections (ABSSSI): practice guidelines for management and care transitions in the emergency department and hospital. J Emerg Med. 2015;48:508-19.

9. Sabbaj A, Jensen B, Browning MA, et al. Soft tissue infections and emergency department disposition: predicting the need for inpatient admission. Acad Emerg Med. 2009;16:1290-7.

10. Schrock JW, Laskey S, Cydulka RK. Predicting observation unit treatment failures in patients with skin and soft tissue infections. Int J Emerg Med. 2008;1:85-90.

11. Peterson D, McLeod S, Woolfrey K, et al. Predictors of failure of empiric outpatient antibiotic therapy in emergency department patients with uncomplicated cellulitis. Acad Emerg Med. 2014;21:526-31.

12. Marwick C, Broomhall J, McCowan C, et al. Severity assessment of skin and soft tissue infections: cohort study of management and outcomes for hospitalized patients. J Antimicrob Chemother. 2011;66:387-97.

13. Eron LJ, Lipsky BA, Low DE, et al. Managing skin and soft tissue infections: expert panel recommendations on key decision points. J Antimicrob Chemother. 2003;52(Suppl 1):i3-17.

14. Wilson SE, Solomkin JS, Le V, et al. A severity score for complicated skin and soft tissue infections derived from phase III studies of linezolid. Am J Surg. 2003;185:369-75.

15. Team CRES. Guidelines on the management of cellulitis in adults. http://www.acutemed.co.uk/ docs/Cellulitis\%20guidelines,\%20CREST,\%2005. pdf. Accessed Sept 9, 2014.

16. Ki V, Rotstein C. Bacterial skin and soft tissue infections in adults: a review of their epidemiology, pathogenesis, diagnosis, treatment and site of care. Can J Infect Dis Med Microbiol. 2008;19:173-84.

17. Marwick C, Rae N, Irvine N, et al. Prospective study of severity assessment and management of acute medical admissions with skin and soft tissue infection. J Antimicrob Chemother. 2012;67:1016-9.

18. Administration FaD. Guidance for industry: acute bacterial skin and skin structure infections. http:// www.fda.gov/downloads/Drugs/.../Guidances/ucm 071185.pdf. Accessed Sept 18, 2014.

19. (ACEP) ACoEM. State of the art: observation units in the emergency department. http://www.acep. org/Clinical-Practice-Management/EmergencyDepartment-Observation-Services/. Accessed 30 May 2018.

20. Dumkow LE, Kenney RM, MacDonald NC, et al. Impact of a multidisciplinary culture follow-up program of antimicrobial therapy in the emergency department. Infect Dis Ther. 2014;3:45-53.

21. Link WA, Barker RJ. Model weights and the foundations of multimodel inference. Ecology. 2006;87:2626-35.

22. Hosmer DW, Hosmer T, Le Cessie S, et al. A comparison of goodness-of-fit tests for the logistic regression model. Stat Med. 1997;16:965-80.

23. Hajian-Tilaki K. Receiver operating characteristic (ROC) curve analysis for medical diagnostic test evaluation. Casp J Intern Med. 2013;4:627-35.

24. Hashem NG, Hidayat L, Berkowitz L, et al. Management of skin and soft-tissue infections at a community teaching hospital using a severity-ofillness tool. J Antimicrob Chemother. 2016;71:3268-75. 
25. Kate Sulham KL, Fan W, Wikler M, Perez A, Good S, Corey R, Lodise T. Severity and costs of acute bacterial skin and skin structure infections by treatment setting: an application of the Eron classification to a real-world database. Montreal: International Society of Pharmacoeconomics and Outcomes Research; 2014.

26. Paterson R, MacLeod DC, Thetford D, et al. Prediction of in-hospital mortality and length of stay using an early warning scoring system: clinical audit. Clin Med. 2006;6:281-4.

27. Ektare V, Khachatryan A, Xue M, et al. Assessing the economic value of avoiding hospital admissions by shifting the management of gram + acute bacterial skin and skin-structure infections to an outpatient care setting. J Med Econ. 2015;18:1092-101.

28. Lodise TP, Fan W, Sulham KA. Hospital admission patterns in adult patients with skin and soft tissue infections: Identification of potentially avoidable hospital admissions through a retrospective database analysis. Hosp Pract. 2015;43:137-43.

29. Jaeschke R. Users' guides to the medical literature: III. How to use an article about a diagnostic test A. Are the results of the study valid? JAMA. 1994;271(5):389. 